\title{
Different Clinicopathologic and Computed Tomography Imaging Characteristics of Primary and Acquired EGFR T790M Mutations in Patients with Non-Small-Cell Lung Cancer
}

\author{
Donghui Hou' \\ Weihua $\mathrm{Li}^{2}$ \\ Sicong Wang ${ }^{3}$ \\ Yao Huang ${ }^{\circ}$ \\ Jianwei Wang' \\ Wei Tang' \\ Lina Zhou' \\ Linlin $\mathrm{Qi}^{1}$ \\ Ning $W_{u^{1,4, *}}$ \\ Shijun Zhaol,*
}

'Department of Diagnostic Radiology, National Cancer Center/National Clinical

Research Center for Cancer/Cancer Hospital, Chinese Academy of Medical Sciences and Peking Union Medical College, Beijing, People's Republic of China;

2Department of Pathology, National Cancer Center/National Clinical Research Center for Cancer/Cancer Hospital, Chinese Academy of Medical Sciences, Beijing, People's Republic of China; ${ }^{3} \mathrm{GE}$ Healthcare, Life Sciences, Beijing, People's Republic of China; ${ }^{4} \mathrm{PET}$-CT Center, National Cancer Center/National Clinical Research Center for Cancer/Cancer Hospital, Chinese Academy of Medical Sciences and Peking Union Medical College, Beijing, People's Republic of China

*These authors contributed equally to this work

Correspondence: Shijun Zhao; Ning Wu Department of Diagnostic Radiology, National Cancer Center/National Clinical Research Center for Cancer/Cancer Hospital, Chinese Academy of Medical Sciences and Peking Union Medical College, No. 17, Panjiayuan Nanli, Beijing, I002I,

People's Republic of China

$\mathrm{Tel}+86-10-8778-8550$;

$\mathrm{Tel} / \mathrm{Fax}+86-10-8778-7523$

Email zsj.200।@I63.com;

cjr.wuning@vip.l63.com
Purpose: Although patients with primary and acquired epidermal growth factor receptor (EGFR) T790M positive non-small-cell lung cancer (NSCLC) respond to osimertinib treatment, the optimal treatment strategy differs for these two groups of patients. This study aimed to compare the clinicopathologic and computed tomography (CT) imaging characteristics between primary and acquired EGFR T790M mutations in patients with NSCLC before treatment.

Patients and Methods: We enrolled two groups of patients with primary or acquired EGFR T790M mutation NSCLC ( $\mathrm{n}=103$ per group) from January 2012 to December 2019. We analyzed their clinicopathologic and CT characteristics and differences between the groups. The groups were further categorized based on $21 \mathrm{~L} 858 \mathrm{R}$ and $19 \mathrm{del}$ to exclude the effect of coexistent mutations.

Results: Primary, compared to acquired, T790M mutation tends to coexist with 21L858R (P $<0.001)$, exhibiting earlier tumor stage $(\mathrm{P}<0.001)$, higher differentiation $(\mathrm{P}=0.029)$, higher proportion of lepidic subtype adenocarcinoma $(\mathrm{P}<0.001)$, and significant associations with some CT features (multiple primary lung cancers, ground-glass opacity, air bronchogram, and vacuole sign [all $\mathrm{P}<0.001]$ ). The combined model, composed of clinicopathologic and conventional CT signature and CT-radiomic signature, showed good discriminative ability with the area under the receiver operating characteristic curve 0.90 and 0.91 in the training and validation datasets, respectively. The $T 790 M$ mutation contributed to these differences independently of coexistent mutations.

Conclusion: We identified clinicopathologic and CT imaging differences between primary and acquired $T 790 M$ mutations. These findings provide insights into developing future personalized $T 790 M$ mutation status-based theranostic strategies.

Keywords: non-small-cell lung cancer, T790M mutation, osimertinib, clinicopathologic characteristic, computed tomography

\section{Introduction}

Non-small-cell lung cancer (NSCLC) accounts for approximately 85\% of all lung cancer cases and is the leading cause of cancer-related deaths worldwide. ${ }^{1}$ Over the last two decades, the discovery of oncogenic alterations and targeted therapeutic agent applications have greatly improved the survival of patients with NSCLC. ${ }^{2}$ Epidermal growth factor receptor (EGFR) mutation is the most common gene alteration, with a frequency of $47.9 \%$ in Asian patients with NSCLC., ${ }^{3,4}$ The 
small deletion in exon 19 and the single amino acid substitution L858R in exon 21 , both of which are activating mutations, account for $85 \%$ of all EGFR mutations. ${ }^{5,6} \mathrm{At}$ present, first- and second-generation tyrosine kinase inhibitors (TKIs), such as gefitinib and afatinib, serve as standard first-line treatments for patients with these activating mutations; ${ }^{7-9}$ however, most patients acquire resistance within 10-14 months after initial treatment. ${ }^{10}$ Approximately half of all resistant tumors develop an EGFR T790M point mutation within exon 20, thereby preventing effective inhibition by EGFR-TKIs. ${ }^{11}$ In addition to the acquired $T 790 M$ mutation, primary $T 790 M$ mutation is occasionally detected in TKI-naive patients with NSCLC during routine mutation testing. ${ }^{12}$ Similar to those with acquired mutations, patients with primary T790M mutation have poor responses to first- or secondgeneration TKIs. ${ }^{13-15}$

Osimertinib has been approved as the front-line therapy for patients with advanced NSCLC with EGFR mutation. It has promising effects in $T 790 M$-positive patients who have progressed from reversible EGFR-TKI treatments $^{16}$ and is also effective in patients with primary T790M mutation. ${ }^{17,18}$ Although patients with primary and acquired EGFR T790M mutation NSCLC respond well to OSIMERTINIB, the optimal treatment strategy differs for these two groups of patients. ${ }^{17-20}$ A sequencing strategy of the first- or second-generation TKIs followed by osimertinib could offer sustained clinical benefit in patients with acquired EGFR T790M mutation NSCLC; ${ }^{19,20}$ however, for patients with primary $T 790 M$ positive NSCLC, osimertinib may be the first-choice TKI. ${ }^{17,18}$ Thus, timely and accurate diagnosis of $7790 M$ mutation is critical for physicians to select appropriate patients for improved treatment outcomes.

Tissue biopsy is considered the standard procedure for molecular genotyping, but it has several limitations, including procedural invasiveness and the risk of falsenegative results due to tumor heterogeneity or low tumor cellularity. Liquid biopsies are an important complement to tissue biopsies, with the plasma circulating tumor DNA (ctDNA) test having been approved for EGFR T790M detection. $^{21}$ However, ctDNA analysis is technically challenging, requiring both sensitivity and accuracy. It is desirable to extract the features of $T 790 M$ mutations from conventional clinical and imaging data to assist existing detection methods; however, little is known regarding the conventional clinical and radiological characteristics of patients with primary $T 790 M$ mutations and how these differ from acquired T790M mutations before TKI targeted therapy.

In this study, we aimed to retrospectively analyze the clinicopathologic features and computed tomography (CT) imaging characteristics of patients with primary and acquired $T 790 M$ mutations identified through routine clinical molecular testing prior to TKI administration, also exploring the distinct differences between them. In addition, we introduced CT radiomics ${ }^{22}$ and examined its usefulness in detecting the T790M mutation.

\section{Materials and Methods}

This study was conducted in accordance with the principles of the Declaration of Helsinki and was approved by the Institutional Ethics Committee of the Cancer Hospital, Chinese Academy of Medical Sciences (No. NCC2017ZDY-03); written informed consent was waived due to anonymous data analysis.

\section{Patients}

In total, the data of 11,957 TKI-naive patients with EGFRpositive NSCLC were retrieved from the Pathology Department data system of the Cancer Hospital, Chinese Academy of Medical Sciences between January 2012 and December 2019. The inclusion criteria were 1) contrastenhanced CT performed within 4 weeks before surgery or biopsy, 2) cancer lesions that could be measured and delineated, and 3) presence of a T790M mutation before treatment or after progressing from first- or secondgeneration TKIs (Supplemental Figure 1). When multiple primary lung cancers were present, the lesion undergoing biopsy was included, and if no direct genetic testing had been conducted on the lung cancer lesion, the largest lung cancer lesion was included. Since the incidence of acquired $T 790 M$ mutations is much higher than that of primary mutations, we only enrolled consecutive patients with acquired $T 790 M$ mutations with the same number of primary mutations in a chronological order.

\section{Pathological Diagnosis and EGFR Mutation Detection}

Histopathology and molecular pathology data were retrieved from the Pathology Department data system in our hospital. Routinely, two experienced pathologists (at least one with more than 20 years of pathological diagnosis experience) performed all histological preparations and analyses. Any disagreement was discussed and 
resolved by a mutual consensus or after consultation with a third pathologist.

\section{Approaches to Obtain Pathological Specimens}

The approaches for obtaining pathological diagnostic specimens are listed in Supplemental Table 1. All patients underwent pathological investigation before TKI-treatment. Patients with an acquired T790M mutation were re-biopsied following resistance to firstor second-generation EGFR-TKIs. The biopsy method was determined by the physician according to the patient's condition, including surgical operation sample, CT-guided lung biopsy, endobronchial ultrasound (EBUS) or bronchofibroscopy biopsy, axilla, supraclavicular or cervical lymph node biopsy, other solid organ (chest wall, liver, adrenal gland) biopsy, plasma ctDNA detection, pleural fluid ctDNA detection, and cerebrospinal fluid ctDNA detection. Eight patients underwent rebiopsy twice or more to confirm their mutation status (four patients underwent plasma circulating tumor DNA detection 2-3 times until testing positive, one underwent US-guided supraclavicular lymph node biopsy, two underwent CT-guided lung biopsy following a T790M mutation-negative plasma ctDNA detection result, and one underwent a CT-guided lung biopsy following a $T 790 M$ mutation-negative pleural fluid ctDNA detection result). If a result was $T 790 M$ mutation-positive, the patient was considered to have $T 790 M$ mutation positivity, and the first $T 790 M$-positive biopsy method was recorded.

\section{Pathological Diagnosis}

Two pathologists diagnosed lung cancer and determined the adenocarcinoma subtype according to the 2011 International Association for the Study of Lung Cancer/ American Thoracic Society/European Respiratory Society international multidisciplinary lung adenocarcinoma criteria. ${ }^{23}$ Pathological types were determined according to predominant pathological components, including acinar, lepidic, papillary, micropapillary, and solid contents. The analysis also included other adenocarcinoma subtype components, which account for more than 5\%, thereby avoiding the omission of components (such as solid type and micropapillary type) that represent a small proportion but have great effects on the patient's prognosis.

\section{EGFR Mutation Detection}

For surgical operative and biopsy specimens, we analyzed $E G F R$ mutations from exons 18 to 21 using commercial
Human EGFR/KRAS Gene Mutation Detection Kits (Beijing, ACCB, Biotech Ltd., China), which were approved by the China Food and Drug Administration for clinical mutation detection with a sensitivity of $1 \%$. The kits consist of a quantitative reverse-transcription polymerase chain reaction platform combining amplification refractory mutation system (ARMS) primers and TaqMan probes. Next-generation sequencing (NGS) was used to detect mutations in the ctDNA of plasma, pleural fluid, and cerebrospinal fluid. These two detection methods have been described in detail in previous studies. ${ }^{18,24}$

\section{Conventional CT Imaging Assessment}

All CT scans were obtained using 64-detector row scanners (LightSpeed VCT, Discovery CT750 HD, Optima CT660, or Revolution CT, General Electric Medical Systems; Toshiba Aquilion, Toshiba Medical Systems; Definition Edge, Siemens Medical Systems) at full inspiration. The CT parameters were as follows: tube voltage, Definition Edge, $100 \mathrm{kVp}$; other scanners, $120 \mathrm{kVp}$; auto mA settings (tube current, 200-350 mA; noise index, 13; pitch, 0.992 or 0.984 ; rotation time, $0.5 \mathrm{~s}$; thickness, $5 \mathrm{~mm}$ ). Reconstruction thickness was 1.25 or $1.0 \mathrm{~mm}$, and the intervals were $0.8 \mathrm{~mm}$ using a standard reconstruction algorithm. The contrast material was iopromide, injected intravenously (iodine concentration, $300 \mathrm{mg} / \mathrm{mL}$ ) at a dose of $80-90 \mathrm{~mL}$ and a flow rate of $2.5 \mathrm{~mL} / \mathrm{s}$; images were obtained $35 \mathrm{~s}$ after intravenous injection. All axial and multiplanar reconstruction $\mathrm{CT}$ images were reviewed in picture archiving and communication system (PACS) using mediastinal (width, $360 \mathrm{HU}$; level, $50 \mathrm{HU}$ ), lung (width, $1500 \mathrm{HU}$; level, -650 HU), and bone (width, 1600 $\mathrm{HU}$; level, $500 \mathrm{HU}$ ) window settings.

Two radiologists (with 5 and 20 years of experience in chest CT, respectively) analyzed chest CT images before cancer treatment. The morphological characteristics of tumor lesions, including the longest and short-axis diameter in the same section of the tumor, the presence of ground-glass opacity (GGO), lobulation, spiculation, air bronchogram, vacuole sign, vessel convergence, pleura abutting, pleural tag, and central or peripheral location, were evaluated. ${ }^{25}$ Spiculation was defined as the presence of linear lines, approximately $2 \mathrm{~mm}$ thick, extending from the tumor margin. ${ }^{26}$ Multiple primary lung cancers were identified by referring to the patient's medical records and pathological reports, and reviewing a series of CT images. ${ }^{27,28}$ The presence of pleural effusion and metastatic sites was also evaluated using abdominal $\mathrm{CT}$, brain 
magnetic resonance imaging, and fluorodeoxyglucose (FDG)-positron emission tomography/CT. All radiologists were blinded to both clinical and histological findings. Disagreements were resolved through consultation with a third radiologist.

\section{CT Radiomic Analysis}

The quantitative CT radiomic workflow is shown in Supplemental Figure 2. All images were resampled to a voxel size of $1 \times 1 \times 1 \mathrm{~mm}^{3}$ using Artificial Intelligence Kit software (AK software; GE Healthcare, Beijing, China). Tumor regions of interest were semi-automatically segmented on processed axial enhanced CT images using ITK-SNAP 3.6 software (http://www.itksnap.org) by a junior radiologist and then validated by a senior radiologist.

In total, 1316 radiomic features were extracted for each lesion from the CT images using AK software based on the open-source Pyradiomics Python package, including 18 firstorder histograms, 24 gray-level co-occurrence matrices, 14 shapes, 14 gray-level dependence matrices, 16 gray-level sizezone matrices, 16 gray-level run-length matrices, 744 wavelets, 5 neighboring gray-tone difference matrices, 186 Gaussian (LoGsiama $=2.0 / 3.0)$, and 279 local binary pattern features. We used two feature selection methods, 1) minimum redundancy maximum relevance and 2) least absolute shrinkage and selection operator, the former of which was first performed to simultaneously select highly predictive but uncorrelated features based on their relevance-redundancy index-based ranking. ${ }^{29}$ Next, least absolute shrinkage and selection operator was used to select the optimized subset of features and evaluate their corresponding coefficients. ${ }^{30}$ The predictive model and rad-score were obtained using 10-fold cross-validation to perform logistic linear regression of the selected features in a linear combination weighted by their respective coefficients, and repeated 10 times.

\section{Statistical Analysis}

Continuous data that fitted the normal distribution are expressed as mean \pm standard deviation, while continuous data that did not fit the normal distribution are expressed as median (range). Categorical data are presented as numbers (percentages). Univariate analyses were performed to assess the difference in clinical, pathological, and CT imaging characteristics between patients with primary and acquired T790M mutations. The Mann-Whitney $U$-test was used for continuous variables and Pearson's chi-square test or Fisher's exact test were used for categorical variables. A multivariate logistic analysis was used to analyze the independent influencing factors that discriminate between primary and acquired T790M mutations; odds ratios (ORs) were also calculated. Significant $(P<0.05)$ variables in the univariate analyses were included in the multivariate analysis following the stepwise selection method.

The quantitative CT radiomic signature or rad-score was calculated by linearly fitting selected radiomic features after weighing them according to their respective coefficient using logistic regression; this was compared using Wilcoxon's rank-sum test.

To determine the additional value of the quantitative CT radiomic signature, compared to conventional clinical and CT imaging features, we developed and compared three multifactor models, namely, clinical signature (identified by multivariate logistic analysis of general clinical, pathologic, and conventional imaging features), radiomic signature, and the combined model. Patients (206 in total) were randomly divided into training and validation datasets at a 7:3 ratio. The area under the receiver operating characteristic curve (AUC), accuracy, sensitivity, and specificity were used to evaluate the performance of the three models in the validation dataset. The calibration curve and Hosmer-Lemeshow test were used to assess the calibration and goodness-of-fit of the combined model. ${ }^{31}$ Decision curve analysis (DCA) was conducted to independently evaluate the clinical value of the three models based on the calculation of the net benefit for patients at each threshold probability. By comparing to all or no strategies, the best model was selected according to the highest calculated net benefit.

In addition, since primary and acquired T790M mutations often coexisted with exon 21 and 19, respectively, we performed a further subgroup analysis of patients with $21 \mathrm{~L} 858 \mathrm{R}$ and $19 \mathrm{del}$ mutations, to verify that the differences between the two groups were not caused by the inherent differences between $21 \mathrm{~L} 858 \mathrm{R}$ and $19 \mathrm{del}$.

All statistical analyses in the present study were performed using R (https://mran.microsoft.com; version 3.5.1) and Python (https://www.python.org; version 3.5.6). Statistical significance was defined as a two-tailed P-value $<0.05$.

\section{Results}

\section{Clinicopathologic and Conventional CT Imaging Characteristics}

Of the 11,957 TKI-naive patients with EGFR-positive NSCLC, 125 had primary T790M mutation, accounting for $1.05 \%$ of the entire EGFR mutation spectrum. After excluding 8 patients who had not undergone CT 
examination in our hospital before treatment, 9 patients who had undergone non-enhanced CT examination, 2 patients who had CT images with heavy artifacts that affected observation, 2 patients who had lesions that were indistinguishable from the surrounding atelectasis, and 1 patient for whom it was difficult to determine the primary tumor, 103 patients with primary $T 790 M$ mutation were included. According to the ratio of $1: 1$ to the primary mutation, 103 eligible patients with acquired T790M mutation detected after first- or second-generation EGFR-TKI treatment were included in a chronological order.

The clinicopathologic characteristics of all patients with EGFR T790M mutation are summarized in Table 1. Among all patients, the median age was 59 years (interquartile range, 52 66 years), $58.25 \%$ were females and $72.33 \%$ were nonsmokers. In this study, patients with a primary T790M mutation were more likely to have multiple primary lung cancers $(\mathrm{P}<$ $0.001)$ and an earlier stage of disease $(\mathrm{P}<0.001)$. The T790M mutation was almost always present in adenocarcinoma (201/ 206), generally coexisting with other mutations in EGFR exons 18,19 , or 21 . Of 103 patients with primary $T 790 M$ mutation, $78(75.73 \%)$ and $18(17.48 \%)$ had $21 L 858 R$ and deletions in exon 19, respectively, whereas 71 (68.93\%) and 32 (31.07\%) acquired $7790 \mathrm{M}$ mutations coexisted with $19 \mathrm{del}$ and $21 \mathrm{~L} 858 \mathrm{R}$, respectively; and rare mutations were relatively common in primary $T 790 M$ mutations ( $\mathrm{P}<0.001$ ). Among the 168 adenocarcinomas for which the differentiation degree was available in the Pathology Department data system, highly differentiated adenocarcinoma had a higher proportion of primary, rather than acquired, T790M mutations (23.96\% vs $8.33 \%, \mathrm{P}=0.029)$. Among the 133 adenocarcinomas for which subtypes were available in the Pathology Department data system, the histological subtypes of the two groups were significantly different $(\mathrm{P}=0.01$, Table 2$)$. Acinar-predominant adenocarcinoma was the most common subtype in both groups; lepidic-predominant adenocarcinoma was found only in the primary $T 790 M$ mutation group, while the proportion of other predominant adenocarcinoma subtypes was higher in the acquired mutation group. Regarding the components of other $>5 \%$ adenocarcinoma subtypes, the presence of a lepidic component $(\mathrm{P}<0.001)$ was more common in primary T790M mutation, while the presence of a solid component $(\mathrm{P}=$ 0.018 ) was more common in acquired mutation.

Initial baseline conventional CT morphologic characteristics are presented in Table 3. Consistent with tumor stage, tumors in the acquired mutation group were larger $(\mathrm{P}<0.001)$, and pleural effusions $(\mathrm{P}=0.001)$ were more common. Among other CT morphological characteristics, the presence of GGO $(\mathrm{P}<0.001)$ and air bronchogram $(\mathrm{P}$ $<0.001)$ were significantly more frequent in patients with primary $T 790 M$ mutation, while pleura attachment $(\mathrm{P}=$ 0.019) was more frequent in patients with acquired $T 790 M$ mutation (Figure 1).

In the multivariable logistic analysis of conventional clinicopathologic and CT morphologic characteristics, multiple primary lung cancers (OR, 0.41; 95\% confidence interval [CI]: $0.14,1.21 ; \mathrm{P}=0.11)$, initial $E G F R$ profile (OR, 0.11; 95\% CI: 0.04, 0.25; P < 0.001), air bronchogram (OR, 0.24; 95\% CI: 0.10, 0.57; P = 0.001), and shortaxis diameter (OR, 1.74; 95\% CI: 1.13, 2.67; $\mathrm{P}=0.011)$ remained as significant factors for discriminating patients with primary and acquired $T 790 M$-mutation. The clinical signature, composed of the remaining features, attained an AUC, accuracy, sensitivity, and specificity of 0.85 (95\% CI: $0.79-0.91), 77.4 \%, 75.0 \%$, and $80.3 \%$, respectively, in the training dataset and 0.85 (95\% CI: 0.76-0.95), 81.7\%, $85.2 \%, 78.8 \%$, respectively, in the validation dataset (Figure 2B and C; Supplemental Table 2).

\section{Analysis of Quantitative CT Radiomic Features}

In total, 1316 quantitative $\mathrm{CT}$ radiomic features were extracted from each lung cancer lesion on enhanced CT images; 16 features with non-zero coefficients were selected. A linear model of the radiomic signature was constructed using logistic regression. The rad-score calculation formula is presented in Supplemental Material 1. Rad-scores were significantly lower in patients with a primary, rather than an acquired, T790M mutation, both in the training and validation datasets (both $\mathrm{P}<0.001$, respectively; Wilcoxon's rank-sum test; Supplemental Figure 3A, B). The quantitative radiomic signature achieved an AUC, accuracy, sensitivity, and specificity of 0.84 (95\% CI: $0.78-0.90), 76.0 \%, 91.8 \%$, and $60.3 \%$, respectively, in the training dataset and 0.86 (95\% CI: $0.77-0.95), 71.7 \%, 86.7 \%$, and $56.7 \%$, respectively, in the validation dataset (Supplemental Table 2).

\section{Combination of Clinicopathologic, Conventional CT Imaging, and Radiomic Signatures}

When combining retained conventional clinicopathologic and CT imaging features with the quantitative radiomic signature for multivariate analysis to identify primary $T 790 M$ mutations (Figure 2A), the AUC was higher than when using 
Table I Clinicopathologic Characteristics of Patients Enrolled in the Study

\begin{tabular}{|c|c|c|c|}
\hline Characteristics & Primary T790M $(n=103)$ & Acquired T790M $(n=103)$ & P-value \\
\hline Age $(y)$ & $59.00(52.00,65.00)$ & $60.00(51.00,66.00)$ & 0.876 \\
\hline Females & $59(57.28)$ & $61(59.22)$ & 0.778 \\
\hline Smoker & $31(30.10)$ & $26(25.24)$ & 0.436 \\
\hline Multiple primary lung cancers & $36(34.95)$ & II (10.68) & $<0.001$ \\
\hline \multicolumn{4}{|l|}{ TNM stage ${ }^{a}$} \\
\hline $\mathrm{I} / \mathrm{II} / \mathrm{III} / \mathrm{IV}$ & $61 / 9 / 17 / 16(59.22 / 8.74 / 16.50 / 15.53)$ & $13 / 20 / 17 / 53(|2.62 / 19.42 / 16.50 / 5| .46)$ & $<0.001$ \\
\hline $\mathrm{TI} / \mathrm{T} 2 / \mathrm{T} 3 / \mathrm{T} 4$ & $56 / 33 / 9 / 5(54.37 / 32.04 / 8.74 / 4.85)$ & $10 / 54 / 27 / I 2(9.71 / 52.43 / 26.21 / 11.65)$ & $<0.001$ \\
\hline $\mathrm{N}_{0} / \mathrm{N}_{1} / \mathrm{N}_{2} / \mathrm{N}_{3}$ & $67 / 11 / 17 / 8(65.05 / 10.68 / 16.50 / 7.77)$ & $34 / 19 / 21 / 29(33.01 / 18.45 / 20.39 / 28.16)$ & $<0.001$ \\
\hline$M_{0} / M_{1}$ & $87 / 16(84.47 / 15.53)$ & $48 / 55(46.60 / 53.40)$ & $<0.001$ \\
\hline Pathological type & & & 0.621 \\
\hline Adenocarcinoma & $101(98.06)$ & $100(97.09)$ & \\
\hline Adenosquamous carcinoma & I (0.97) & $3(2.91)$ & \\
\hline Squamous carcinoma & I (0.97) & $0(0.00)$ & \\
\hline Differentiation of adenocarcinoma ${ }^{b}$ & & & 0.029 \\
\hline Highly & $23(23.96 \%)$ & $6(8.33 \%)$ & \\
\hline Moderately & 39 (40.62\%) & $36(50.00 \%)$ & \\
\hline Poorly & 34 (35.42\%) & $30(41.67 \%)$ & \\
\hline Sensitizing EGFR mutations & & & $<0.001$ \\
\hline Exon 19 deletions & $18(17.48)$ & 71 (68.93) & \\
\hline $2 I L 858 R$ & $78(75.73)$ & $32(31.07)$ & \\
\hline Others ${ }^{c}$ & $7(6.80)$ & $0(0.00)$ & \\
\hline
\end{tabular}

Notes: Data are numbers of patients, with percentages in parentheses or medians; interquartile ranges are in parentheses. EGFR, epidermal growth factor receptor; TNM,

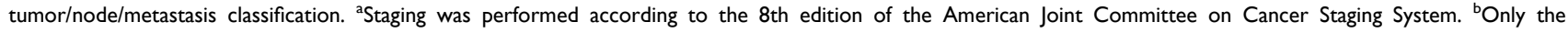

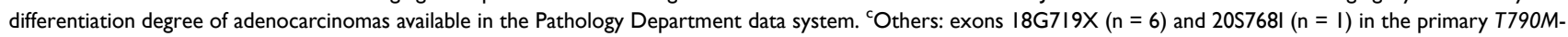
mutant group.

conventional clinical and CT imaging features only, thereby yielding an AUC, accuracy, sensitivity, and specificity of 0.90 (95\% CI, 0.85-0.95), 83.6\%, 83.6\%, and 83.6\%, respectively, in the training dataset and 0.91 (95\% CI: $0.83-0.98), 85.0 \%$, $82.0 \%$, and $92.0 \%$, respectively, in the validation dataset (Figure 2B and C; Supplemental Table 2). The HosmerLemeshow test for the final multi-factor logistic models yielded P-values of 0.677 and 0.352 in the training and validation datasets, respectively, indicating good calibration power. The calibration curve of the combined model is shown in Figure 2D and E.

According to the decision curve, the combined model demonstrated better primary and acquired T790M-mutant discrimination, with more areas shown in the validation cohort than in the conventional clinical and CT imaging signature or quantitative radiomic signature alone (Figure 3 ).

\section{I L858 and I9del-Based Subgroup Analysis}

The results of clinicopathologic and CT imaging characteristics in 21L858 and 19del-based subgroup analysis are shown in Supplemental Tables 3-4. In the 21L858R and $19 \mathrm{del}$ subgroups, we found that patients with a primary T790M mutation had an earlier disease stage $(\mathrm{P}=0.003$, $\mathrm{P}=0.001$, respectively $)$, GGO $(\mathrm{P}=0.031, \mathrm{P}=0.043$, respectively), air bronchogram ( $\mathrm{P}=0.021, \mathrm{P}=0.021$, respectively), and lepidic components ( $\mathrm{P}=0.004, \mathrm{P}=0.01$, respectively). Patients with a primary, compared to an acquired, T790M mutation had multiple primary lung cancers $(\mathrm{P}=0.01)$ in the $21 L 858 R$ subgroup. Although other clinicopathologic and CT features mentioned above were not significant owing to the small number of patients, they still showed the same distribution tendency in subgroup analyses.

\section{Discussion}

We retrospectively analyzed and compared the clinical, pathological, and CT features of patients with primary and acquired $T 790 M$ mutation before treatment, also assessing the prevalence of primary T790M mutations in patients with EGFR-positive NSCLC using routine detection methods. Our study suggests that primary T790M 
Table 2 Histological Subtypes and Components of T790M Mutations ${ }^{\mathrm{a}}$

\begin{tabular}{|c|c|c|c|}
\hline Variable & Primary T790M $(n=87)$ & Acquired T790M $(n=46)$ & P-value \\
\hline \multicolumn{3}{|l|}{ Histological subtype } & 0.01 \\
\hline Acinar-predominant adenocarcinoma & $53(60.92)$ & $30(65.22)$ & \\
\hline Lepidic-predominant adenocarcinoma & $14(16.09)$ & $0(0.00)$ & \\
\hline Papillary-predominant adenocarcinoma & $17(19.54)$ & $13(28.26)$ & \\
\hline Micropapillary-predominant adenocarcinoma & $\mathrm{I}(\mathrm{I.I5)}$ & $\mathrm{I}(2.17)$ & \\
\hline Solid-predominant adenocarcinoma & I (I.15) & $2(4.35)$ & \\
\hline Other subtypes & $\mathrm{I}(\mathrm{I} .15)$ & $0(0.00)$ & \\
\hline \multicolumn{4}{|l|}{ Histological component (>5\%) } \\
\hline Presence of acinar component & $80(91.95)$ & $38(82.61)$ & 0.105 \\
\hline Presence of lepidic component & $44(50.57)$ & $5(10.87)$ & $<0.001$ \\
\hline Presence of papillary component & $55(63.22)$ & $28(60.87)$ & 0.79 \\
\hline Presence of micropapillary component & $25(28.74)$ & $19(41.30)$ & 0.143 \\
\hline Presence of solid component & $9(10.34)$ & $12(26.09)$ & 0.018 \\
\hline
\end{tabular}

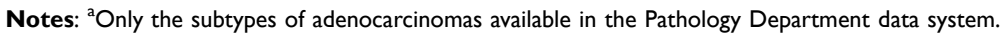

Table 3 Conventional Computed Tomography (CT) Imaging Characteristics Before Treatment

\begin{tabular}{|c|c|c|c|}
\hline Characteristics & Primary T790M $(n=103)$ & Acquired T790M $(n=103)$ & P-value \\
\hline Multiple primary lung cancers & $36(34.95)$ & II (I0.68) & $<0.001$ \\
\hline Location & & & 0.082 \\
\hline Central & $8(7.77)$ & $16(15.53)$ & \\
\hline Peripheral & $95(92.23)$ & 87 (84.47) & \\
\hline Long-axis diameter $(\mathrm{cm})$ & $2.30(1.62,2.98)$ & $3.30(2.50,4.18)$ & $<0.001$ \\
\hline Short-axis diameter $(\mathrm{cm})$ & $1.80(1.30,2.20)$ & $2.50(1.82,3.30)$ & $<0.001$ \\
\hline Presence of GGO & $54(52.43)$ & $23(22.33)$ & $<0.001$ \\
\hline Spiculated sign & $63(61.17)$ & $59(57.28)$ & $0.57 I$ \\
\hline Air bronchogram & $60(58.25)$ & $28(27.18)$ & $<0.001$ \\
\hline Vacuole sign & $23(22.33)$ & II (10.68) & 0.024 \\
\hline Lobulation sign & $90(87.38)$ & $88(85.44)$ & 0.684 \\
\hline Vessel convergence $^{\mathrm{a}}$ & 15 (15.79) & 18 (20.69) & 0.391 \\
\hline Pleura attachment ${ }^{\mathrm{a}}$ & 37 (38.95) & $49(56.32)$ & 0.019 \\
\hline Pleural indentation $^{\mathrm{a}}$ & $39(4 \mid .05)$ & $34(39.08)$ & 0.786 \\
\hline Pleural effusion & $4(3.88)$ & $20(19.42)$ & 0.001 \\
\hline
\end{tabular}

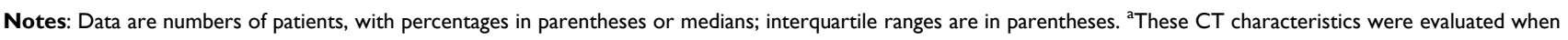
the tumor was located peripherally.

Abbreviation: GGO, ground-glass opacity.

mutation is rare $(1.05 \%)$ in mainland Chinese patients with EGFR-positive NSCLC detected by routine mutation testing, which is consistent with the results of a previous study from another center in mainland China $(1.07 \%$ in patients with EGFR-positive NSCLC $)^{17}$ and lower than those of a study in Taiwan $(25.2 \%$ in patients with NSCLC detected by matrix-assisted laser desorption ionizationtime of flight mass spectrometry and $2.8 \%$ detected by NGS) ${ }^{15}$ and another study of non-Asian patients $(6.9 \%$ in patients with EGFR-positive NSCLC). ${ }^{32}$ Although T790M 


\section{A}

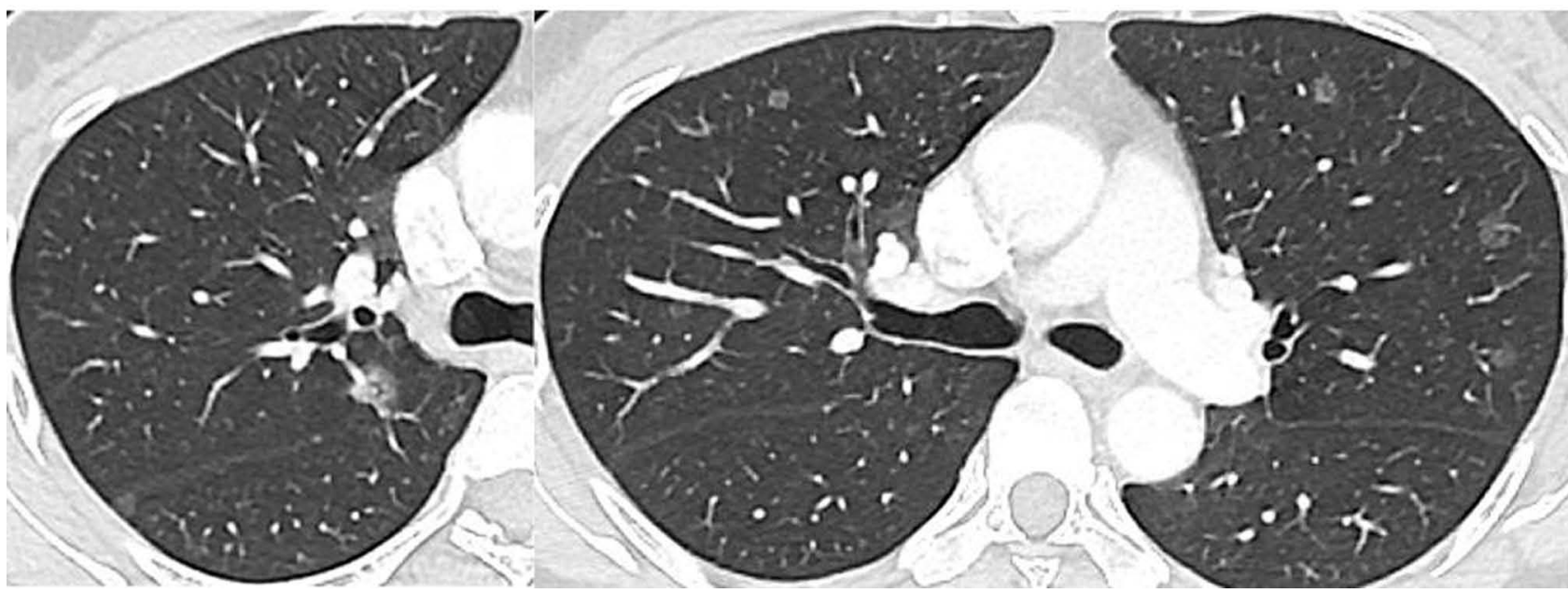

Figure I Images of a 48-year-old female tyrosine kinase inhibitor (TKI)-naive patient with T790M mutation-positive non-small-cell lung cancer (NSCLC). (A) Computed tomography (CT) image in the axial lung window setting showing a $1.2-\mathrm{cm}$ lung nodule in the right upper lobe under the horizontal fissure, with an internal air bronchogram. (B) Axial CT image showing multiple ground-glass nodules in the bilateral lungs.

detection rates increase with higher sensitivity methods, ${ }^{33,34}$ false-positive rates should be considered in clinical molecular testing, especially for samples with low DNA quality and quantity and/or formalin-fixed and paraffin-embedded-derived artifacts. ${ }^{35,36}$ Here, we used two routine detection methods, ARMS and NGS, to accurately diagnose primary T790M mutation, in line with the current clinical reality. Moreover, primary and acquired T790M mutations coexisted with $21 L 858 R$ and $19 \mathrm{del}$, respectively, while rare mutations were relatively common in primary, rather than in acquired, T790M mutations, which was consistent with the results of a previous study. ${ }^{17}$

In our study, patients with primary $T 790 M$ mutation exhibited an earlier stage of disease than those with an acquired mutation, which seems to be "inappropriate" but corresponds to the clinical reality. Preclinical studies found that the $T 790 M$ mutation was associated with more indolent growth and a growth disadvantage in the absence of TKI selection in in vitro models. ${ }^{37,38}$ In several other small clinical studies, patients with the primary T790M mutation also exhibited an early disease stage. ${ }^{39-41}$ As mentioned above, primary and acquired $T 790 M$ mutation coexisted with $21 L 858 R$ and $19 \mathrm{del}$, respectively; the different molecular characteristics of these two coexistent mutations also led to different clinicopathologic and radiological characteristics. ${ }^{9,42}$ The clinical effect of EGFR mutation subtypes was analyzed in a large cohort in Japan, and the results indicated that a deletion in exon 19 correlated with an advanced pathological stage (trend $\mathrm{P}<0.001$ ), a more aggressive phenotype, and a poorer prognosis than $21 L 858 R .^{43}$ By comparing the histological subtype in the two groups, we found that the low-grade malignant lepidic subtype was more abundant and high-grade malignant solid and micropapillary subtypes were less abundant in primary $T 790 M$ mutations than in the acquired group. This may also explain why primary T790M mutations were associated with earlier pathological stages than the acquired group. Moreover, early-stage patients undergoing radical surgery generally do not routinely receive other adjuvant treatments, such as chemotherapy or targeted therapy. In other words, patients who are traceable to targeted therapies and develop resistant $T 790 M$ mutations tend to have a relatively advanced tumor.

Some patients cannot tolerate surgical biopsy; however, liquid DNA detection is prone to false negatives (8 patients T790M mutation-negative according to liquid DNA were finally found to have the $T 790 M$ mutation in this study), and tumor heterogeneity and current biopsy methods appear incomplete. Therefore, we attempted to use CT features to assist the diagnosis of gene mutations. Previous studies have shown associations among CT features, genetic mutations, and histological subtypes in lung adenocarcinoma. ${ }^{44-48}$ Multiple primary lung cancers are more common in adenocarcinomas and contain GGO. ${ }^{44,45} \mathrm{GGO}$ is significantly more common in lepidic-predominant adenocarcinomas than in other histological subtypes ${ }^{46}$ and is more common in EGFR $21 L 858 R$ mutation adenocarcinomas than in $19 \mathrm{del} .{ }^{47}$ Furthermore, air bronchogram and vacuole sign are features of lepidic- 
$\dot{A}$ Points

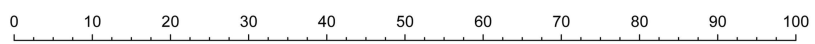

Multiple primary lung cancers

Initial EGFR profile

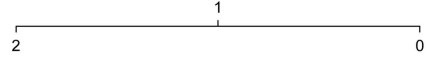

Air bronchogram

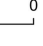

Short axis diameter

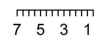

Rad score

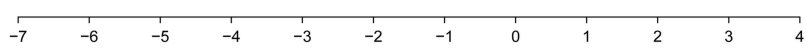

Total Points

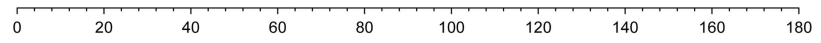

Risk

\section{B}

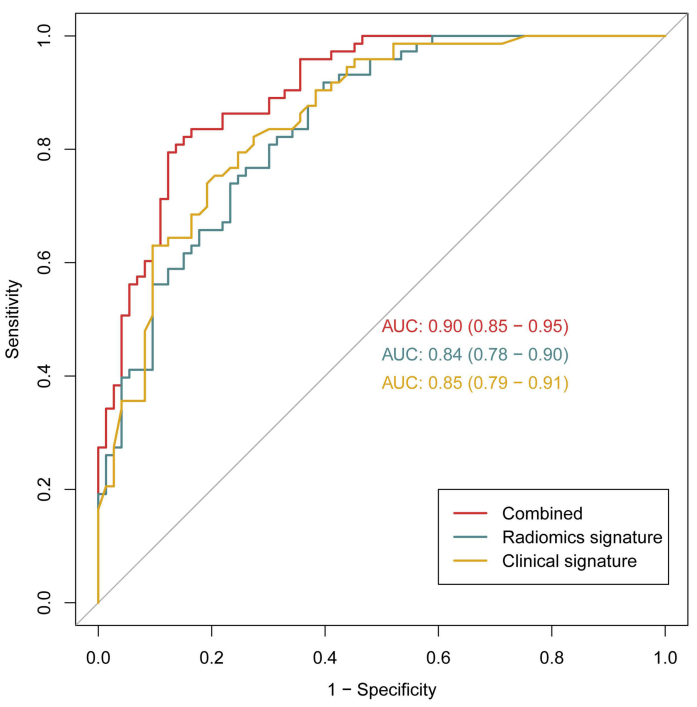

D

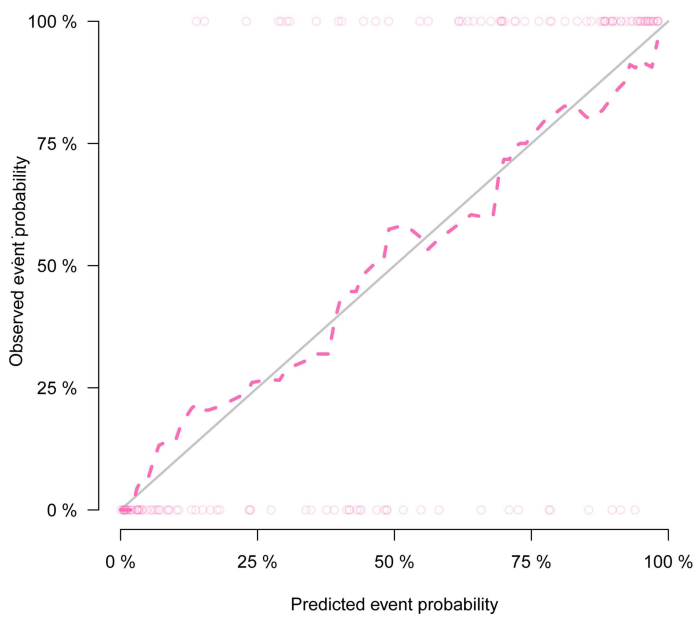

$\begin{array}{lll}0.1 & 0.4 & 0.9\end{array}$

C

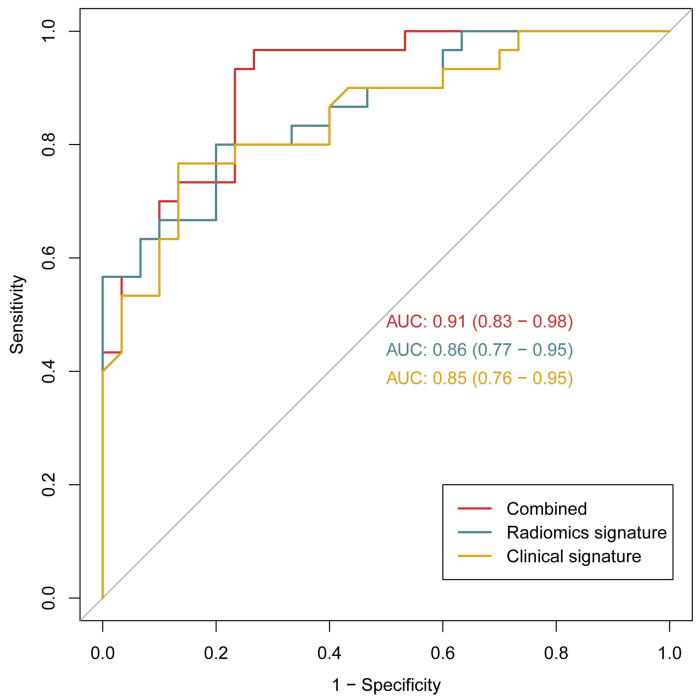

E

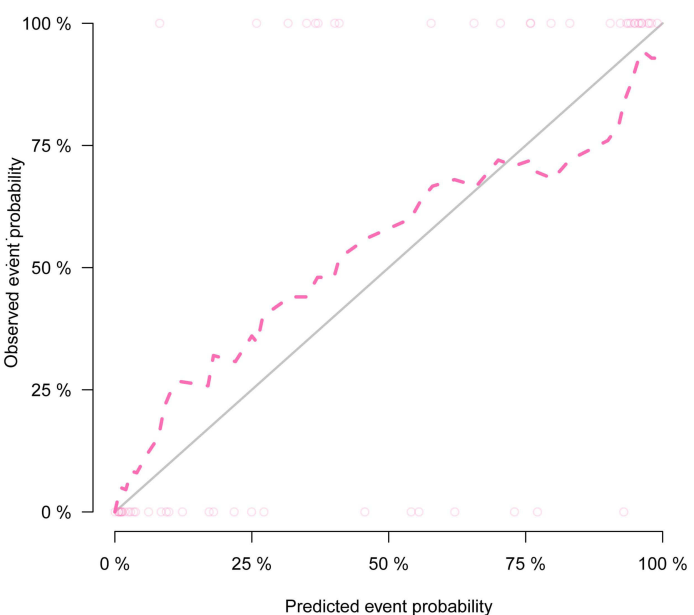

Figure 2 A combined model identifying primary and acquired T790M mutations. (A) Combined model identifying both primary and acquired EGFR T790M mutations. (B and C) Receiver operating characteristic curves for the computed tomography (CT) radiomic signature, routine clinical signature, and combined model in training (B) and validation (C) cohorts. (D and E) Calibration curves of the combined model in the training (D) and validation (E) cohorts. AUC, area under the receiver operating characteristic curve. 


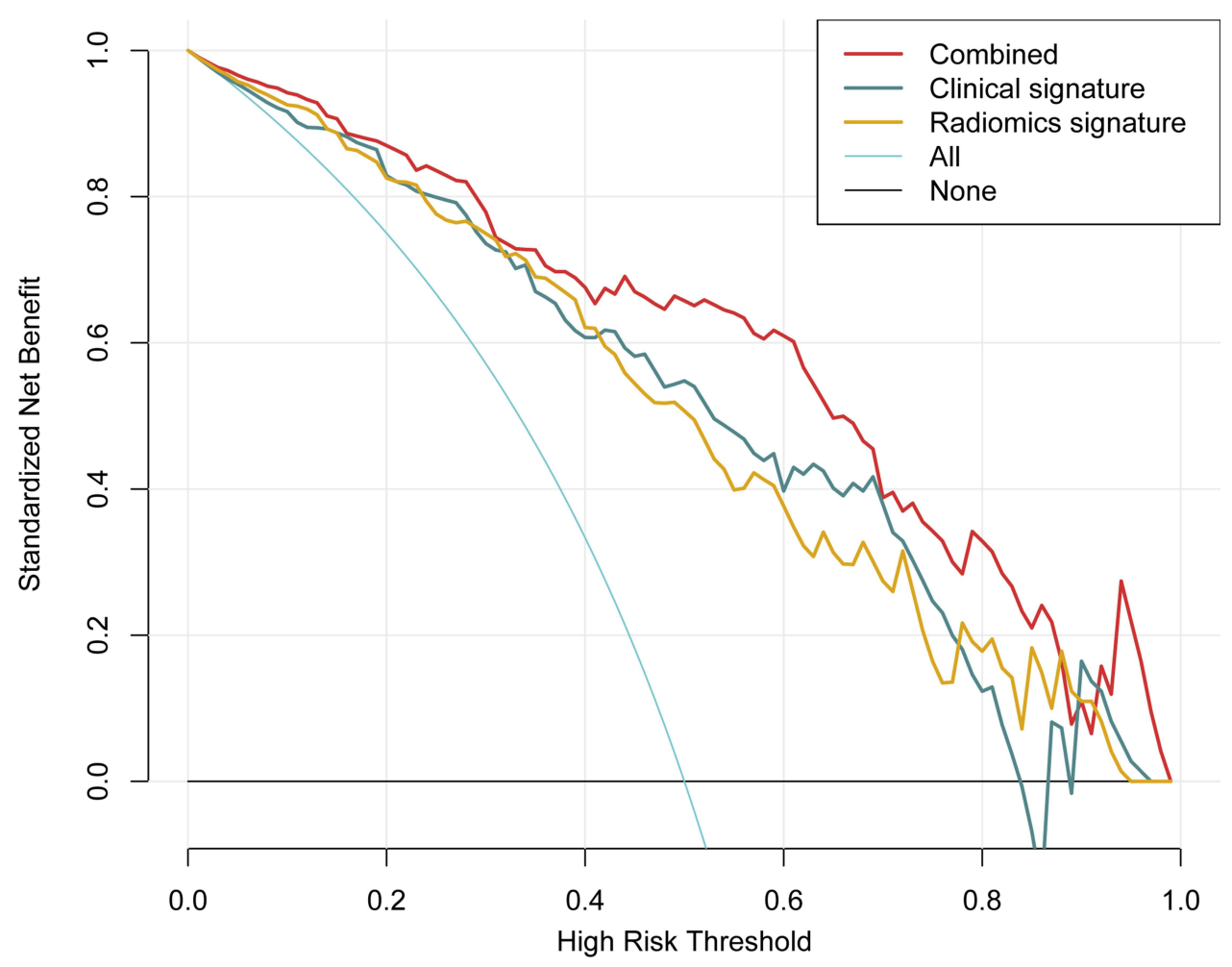

Cost:Benefit Ratio

Figure 3 Decision curve analysis for the combined model in the validation cohort. The y-axis represents the net benefit. The light purple line represents the hypothesis that all patients have the primary T790M mutation; the black line represents the hypothesis that all patients have the acquired T790M mutation. The combined model (red in the figure) improves the performance of T790M mutation prediction with more areas than the computed tomography (CT) radiomic or clinical signatures alone.

dominant histological subtypes and are seen less commonly in other patterns because the solid-density type would obscure the bronchi. ${ }^{42}$ This is the first study to compare the initial CT characteristics between primary and acquired $T 790 \mathrm{M}$ mutations in NSCLC. We observed that patients with primary, rather than acquired, $T 790 M$ mutations had multiple primary lung cancers, GGO, air bronchogram, and vacuole sign in CT images. These findings remained significant following subgroup analyses of $21 \mathrm{~L} 858$ and $19 \mathrm{del}$ or exhibited the same distribution trend but did not achieve statistical significance owing to the small number of patients. These CT features corresponded to earlier stages in patients with a primary T790M mutation and were consistent with indolent growth characteristics.

Finally, we introduced CT radiomics, which relies solely on conventional medical images and is known to be comprehensive, non-invasive, and a quantitative evaluator of tumor heterogeneity. ${ }^{22,48}$ The CT radiomic signature was found to provide additional value in identifying T790M mutations. Similar to previous studies, most of the radiomic features retained through screening were high-order texture parameters describing the complexity, degree of change, and thickness of the lesion microstructure. Although radiomics has not been widely used in the clinic, many studies have shown its excellent performance in tumor diagnosis and efficacy prediction. ${ }^{49,50}$ As multidisciplinary overlap is an inexorable trend in modern medicine, we expect the widespread adoption of a standardized radiomic procedure.

Our study has several limitations. First, as the incidence of primary $T 790 M$ mutations is much lower than that of acquired mutations, it appears unreasonable to include patients with acquired mutations under any criteria. We included patients with acquired T790M mutations over a continuous period, as we believe this reflects the actual clinical situation. Second, although our results were obtained by two experienced chest radiologists, the conventional CT feature assessment was subjective. Third, the proportion of multiple primary lung cancers may be higher than expected because not all cases are pathologically diagnosed. However, it is not feasible for multiple primary lung cancer diagnoses 
to rely solely on pathological diagnosis. High-resolution CT is an important diagnostic technique for diagnosing multiple primary lung cancers with high accuracy. Fourth, spatial heterogeneity of the tumor may influence $T 790 M$ mutation detection. Fifth, this was a preliminary exploratory singlecenter study, and the primary T790M mutation rate is relatively low. Although data from the National Cancer Center of China concerning eight consecutive years was included, the number of patients was still small. Prospective, multicenter research is needed for further verification.

\section{Conclusion}

Primary $T 790 M$ mutations usually coexist with $21 L 858 R$ and are associated with early tumor stage, high differentiation, and a high proportion of lepidic subtype adenocarcinoma, also significantly associated with some CT features, such as multiple primary lung cancers, GGO, air bronchogram, vacuole sign, and quantitative radiomic features. The T790M mutation contributes to these differences independently of its accompanying mutations. These findings provide useful information for T790M mutation status-based personalized diagnosis and treatment strategies when patients cannot tolerate surgery or the specimen is not sufficient for genetic testing following pathological examination.

\section{Abbreviations}

ARMS, amplification refractory mutation system; AUC, area under the receiver operating characteristic curve; ctDNA, circulating tumor DNA; CT, computed tomography; DCA, decision curve analysis; EBUS, endobronchial ultrasound; EGFR, epidermal growth factor receptor; FDG, fluorodeoxyglucose; GGO, ground-glass opacity; NGS, next-generation sequencing; NSCLC, non-smallcell lung cancer; OR, odds ratio; PACS, picture archiving and communication system; PFS, progression-free survival; TKIs, tyrosine kinase inhibitors.

\section{Data Sharing Statement}

The data and material are available from the corresponding author (Shijun Zhao) upon reasonable request.

\section{Ethics Statement}

This study was conducted in accordance with the principles of the Declaration of Helsinki and was approved by the Institutional Ethics Committee of the Cancer Hospital, Chinese Academy of Medical Sciences (No. NCC2017ZDY-03); written informed consent was waived due to anonymous data analysis.

\section{Acknowledgments}

This work was supported by the National Key R\&D Program of China (No. 2017YFC1308700), CAMS Innovation Fund for Medical Sciences (No. 2017-I2M-1-005, 2019-I2M -2-002), National Natural Science Foundation of China (No. 81771830), and Beijing Municipal Science and Technology Project (No. Z201100005620002).

\section{Disclosure}

The authors report no conflicts of interest in this work.

\section{References}

1. Sung H, Ferlay J, Siegel RL, et al. Global cancer statistics 2020: GLOBOCAN estimates of incidence and mortality worldwide for 36 cancers in 185 countries. CA Cancer J Clin. 2021;71:209-249. doi: $10.3322 /$ caac. 21660

2. Sankar K, Gadgeel SM, Qin A. Molecular therapeutic targets in non-small cell lung cancer. Expert Rev Anticancer Ther. 2020;20:647-661. doi:10.1080/14737140.2020.1787156

3. Castellanos E, Feld E, Horn L. Driven by mutations: the predictive value of mutation subtype in EGFR-mutated non-small cell lung cancer. $J$ Thorac Oncol. 2017;12:612-623. doi:10.1016/j. jtho.2016.12.014

4. Dearden S, Stevens J, Wu YL, Blowers D. Mutation incidence and coincidence in non small-cell lung cancer: meta-analyses by ethnicity and histology (mutMap). Ann Oncol. 2013;2:2371-2376. doi:10.1093/annonc/mdt205

5. Lynch TJ, Bell DW, Sordella R, et al. Activating mutations in the epidermal growth factor receptor underlying responsiveness of non-small-cell lung cancer to gefitinib. $N$ Engl $J$ Med. 2004;350:2129-2139. doi:10.1056/NEJMoa040938

6. Gazdar AF. Activating and resistance mutations of EGFR in non-small-cell lung cancer: role in clinical response to EGFR tyrosine kinase inhibitors. Oncogene. 2009;28:S24-S31. doi:10.1038/ onc. 2009.198

7. Cataldo VD, Gibbons DL, Pérez-Soler R, Quintás-Cardama A Treatment of non-small-cell lung cancer with erlotinib or gefitinib. N Engl J Med. 2011;364:947-955. doi:10.1056/NEJMct0807960

8. Shi YK, Wang L, Han BH, et al. First-line icotinib versus cisplatin/ pemetrexed plus pemetrexed maintenance therapy for patients with advanced EGFR mutation-positive lung adenocarcinoma (CONVINCE): a Phase 3, open-label, randomized study. Ann Oncol. 2017;28:2443-2450. doi:10.1093/annonc/mdx359

9. Yang JC, Wu YL, Schuler M, et al. Afatinib versus cisplatin-based chemotherapy for EGFR mutation-positive lung adenocarcinoma (LUX-Lung 3 and LUX-Lung 6): analysis of overall survival data from two randomized, phase 3 trials. Lancet Oncol. 2015;16:141-151. doi:10.1016/S1470-2045(14)71173-8

10. Lim SM, Syn NL, Cho BC, Soo RA. Acquired resistance to EGFR targeted therapy in non-small cell lung cancer: mechanisms and therapeutic strategies. Cancer Treat Rev. 2018;65:1-10. doi:10.1016/j.ctrv.2018.02.006

11. Yu HA, Arcila ME, Rekhtman N, et al. Analysis of tumor specimens at the time of acquired resistance to EGFR-TKI therapy in 155 patients with EGFR-mutant lung cancers. Clin Cancer Res. 2013;19:2240-2247. doi:10.1158/1078-0432.CCR-12-2246

12. Chen LY, Molina-Vila MA, Ruan SY, et al. Coexistence of EGFR $T 790 M$ mutation and common activating mutations in pretreatment non-small cell lung cancer: a systematic review and meta-analysis. Lung Cancer. 2016;94:46-53. doi:10.1016/j.lungcan.2016.01.019 
13. Yu HA, Arcila ME, Hellmann MD, Kris MG, Ladanyi M, Riely GJ. Poor response to erlotinib in patients with tumors containing baseline EGFR T790M mutations found by routine clinical molecular testing. Ann Oncol. 2014;5:423-428. doi:10.1093/annonc/mdt573

14. Rosell R, Molina MA, Costa C, et al. Pretreatment EGFR T790M mutation and BRCA1 mRNA expression in erlotinib-treated advanced non-smallcell lung cancer patients with EGFR mutations. Clin Cancer Res. 2011;17:1160-1168. doi:10.1158/1078-0432.CCR-10-2158

15. Su KY, Chen HY, Li KC, et al. Pretreatment epidermal growth factor receptor $(E G F R)$ T790M mutation predicts shorter EGFR tyrosine kinase inhibitor response duration in patients with non-small-cell lung cancer. $J$ Clin Oncol. 2012;30:433-440. doi:10.1200/ JCO.2011.38.3224

16. Jänne PA, Yang JC, Kim DW, et al. AZD9291 in EGFR inhibitor-resistant non-small-cell lung cancer. $N$ Engl $J$ Med. 2015;372:1689-1699. doi:10.1056/NEJMoa1411817

17. Wang S, Yan B, Zhang Y, et al. Different characteristics and survival in non-small cell lung cancer patients with primary and acquired EGFR T790M mutation. Int $J$ Cancer. 2019;144:2880-2886. doi:10.1002/ijc.32015

18. Li W, Qiu T, Guo L, et al. Primary and acquired EGFR T790M-mutant NSCLC patients identified by routine mutation testing show different characteristics but may both respond to osimertinib treatment. Cancer Lett. 2018;423:9-15. doi:10.1016/j.canlet.2018.03.005

19. Hochmair MJ, Morabito A, Hao D. Sequential afatinib and osimertinib in patients with EGFR mutation-positive non-small-cell lung cancer: final analysis of the GioTag study. Future Oncol. 2020;16:2799-2808. doi:10.2217/fon-2020-0740

20. Shah R, Lester JF. Tyrosine kinase inhibitors for the treatment of EGFR Mutation-positive non-small-cell lung cancer: a clash of the generations. Clin Lung Cancer. 2020;21:e216-e228. doi:10.1016/j. cllc.2019.12.003

21. Planchard D, Popat S, Kerr K, et al. Metastatic non-small cell lung cancer: ESMO Clinical Practice Guidelines for diagnosis, treatment and follow-up. Ann Oncol. 2018;29:iv192-iv237. doi:10.1093/ annonc/mdy275

22. Lambin P, Rios-Velazquez E, Leijenaar R, et al. Radiomics: extracting more information from medical images using advanced feature analysis. Eur $J$ Cancer. 2012;48:441-446. doi:10.1016/j. ejca.2011.11.036

23. Travis WD, Brambilla E, Noguchi M, et al. International association for the study of lung cancer/american thoracic society/European respiratory society international multidisciplinary classification of lung adenocarcinoma. $J$ Thorac Oncol. 2011;6:244-285. doi:10.1097/JTO.0b013e318206a221

24. Shan L, Wang Z, Guo L, et al. Concurrence of EGFR amplification and sensitizing mutations indicate a better survival benefit from EGFR-TKI therapy in lung adenocarcinoma patients. Lung Cancer. 2015;89:337-342. doi:10.1016/j.lungcan.2015.06.008

25. Liu Y, Kim J, Qu F, et al. CT features associated with epidermal growth factor receptor mutation status in patients with lung adenocarcinoma. Radiology. 2016;280:271-280. doi:10.1148/ radiol.2016151455

26. Zwirewich CV, Vedal S, Miller RR, Müller NL. Solitary pulmonary nodule: high-resolution $\mathrm{CT}$ and radiologic-pathologic correlation. Radiology. 1991;179:469-476. doi:10.1148/radiology.179.2.2014294

27. Zhou LN, Wu N, Zhao SJ, et al. HRCT features differentiate synchronous multiple primary lung adenocarcinomas from intrapulmonary metastases. Zhonghua Zhong Liu Za Zhi. 2020;42:449-455. doi:10.3760/cma.j.cn112152-20200227-00126

28. Zhang Y, Li G, Li Y, et al. Imaging features suggestive of multiple primary lung adenocarcinomas. Ann Surg Oncol. 2020;27:2061-2070. doi:10.1245/s10434-019-08109-w

29. Ding C, Peng H. Minimum redundancy feature selection from microarray gene expression data. $J$ Bioinform Comput Biol. 2005;3:185-205. doi:10.1142/S0219720005001004
30. Tibshirani R. Regression shrinkage and selection via the lasso: a retrospective. $J \quad R \quad$ Stat Soc Series $B$ Stat Methodol. 2011;73:273-282. doi:10.1111/j.1467-9868.2011.00771.x

31. Kramer AA, Zimmerman JE. Assessing the calibration of mortality benchmarks in critical care: the Hosmer-Lemeshow test revisited. Crit Care Med. 2007;35:2052-2056. doi:10.1097/01. CCM.0000275267.64078.B0

32. Arrieta O, Cardona AF, Corrales L, et al. The impact of common and rare $E G F R$ mutations in response to $E G F R$ tyrosine kinase inhibitors and platinum-based chemotherapy in patients with non-small cell lung cancer. Lung Cancer. 2015;87:169-175. doi:10.1016/j. lungcan.2014.12.009

33. Fujita Y, Suda K, Kimura H, et al. Highly sensitive detection of EGFR T790M mutation using colony hybridization predicts favorable prognosis of patients with lung cancer harboring activating EGFR mutation. $J$ Thorac Oncol. 2012;7:1640-1644. doi:10.1097/ JTO.0b013e3182653d7f

34. Lavdovskaia ED, Iyevleva AG, Sokolenko AP, et al. EGFR T790M mutation in TKI-naïve clinical samples: frequency, tissue mosaicism, predictive value and awareness on artifacts. Oncol Res Treat. 2018;41:634-642. doi:10.1159/000491441

35. Iwama E, Takayama K, Harada T, et al. Highly sensitive and quantitative evaluation of the EGFR T790M mutation by nanofluidic digital PCR. Oncotarget. 2015;6:20466-20473. doi:10.18632/oncotarget.4058

36. Ye X, Zhu ZZ, Zhong L, et al. High T790M detection rate in TKI-naive NSCLC with EGFR sensitive mutation: truth or artifact? J Thorac Oncol. 2013;8:1118-1120. doi:10.1097/ JTO.0b013e31829f691f

37. Chmielecki J, Foo J, Oxnard GR, et al. Optimization of dosing for $E G F R$-mutant non-small cell lung cancer with evolutionary cancer modeling. Sci Transl Med. 2011;3:90ra59. doi:10.1126/ scitranslmed.3002356

38. Kawamura T, Kenmotsu H, Omori S, et al. Clinical factors predicting detection of T790M mutation in rebiopsy for EGFR-mutant non-small-cell lung cancer. Clin Lung Cancer. 2018;19:e247-e252. doi:10.1016/j.cllc.2017.07.002

39. Hashida S, Soh J, Toyooka S, et al. Presence of the minor EGFR $T 790 M$ mutation is associated with drug-sensitive EGFR mutations in lung adenocarcinoma patients. Oncol Rep. 2014;32:145-152. doi:10.3892/or.2014.3197

40. Sakashita S, Shiba-Ishii A, Murata Y, et al. Case report of three $E G F R$ TKI naïve lung adenocarcinoma containing double EGFR mutations (L858R/T790M or Exon 19 Deletion/T790M); Comparing genetic information and histology. Pathol Res Pract. 2018;214:1224-1230. doi:10.1016/j.prp.2018.05.016

41. Wang X, Li X, Guo H, et al. Highly sensitive droplet digital PCR method for detection of de novo EGFR T790M mutation in patients with non-small cell lung cancer. Onco Targets Ther. 2020;13:10621-10630. doi:10.2147/OTT.S267677

42. Yotsukura M, Yasuda H, Shigenobu T, et al. Clinical and pathological characteristics of EGFR mutation in operable early-stage lung adenocarcinoma. Lung Cancer. 2017;109:45-51. doi:10.1016/j. lungcan.2017.04.014

43. Suda K, Mitsudomi T, Shintani Y, et al. Japanese Joint Committee of Lung Cancer Registry. Clinical impacts of EGFR mutation status: analysis of 5780 surgically resected lung cancer cases. Ann Thorac Surg. 2021;111:269-276. doi:10.1016/j.athoracsur.2020.05.041

44. Weng CF, Chen PJ, Tseng AH, Huang SH, Hsin-Chun H. Unusual synchronous double primary treatment-naïve lung adenocarcinoma harboring $T 790 M$ and $L 858 R$ mutations in early-stage lung cancer. World J Surg Oncol. 2019;17:2-6. doi:10.1186/s12957-019-1688-3

45. Bai Y, Qiu J, Shang X, et al. Differential diagnosis and cancer staging of a unique case with multiple nodules in the lung - lung adenocarcinoma, metastasis of colon adenocarcinoma, and colon adenocarcinoma metastasizing to lung adenocarcinoma. Thorac Cancer. 2015;6:363-367. doi:10.1111/1759-7714.12173 
46. Sun F, Xi J, Zhan C, et al. Ground glass opacities: imaging, pathology, and gene mutations. $J$ Thorac Cardiovasc Surg. 2018;156:808-813. doi:10.1016/j.jtcvs.2018.02.110

47. Yang Y, Yang Y, Zhou X, et al. EGFR L858R mutation is associated with lung adenocarcinoma patients with dominant ground-glass opacity. Lung Cancer. 2015;87:272-277. doi:10.1016/j.lungcan.2014.12.016

48. Gillies RJ, Kinahan PE, Hricak H. Radiomics: images are more than pictures, they are data. Radiology. 2016;278:563-577. doi:10.1148/ radiol.2015151169
49. Ji Y, Qiu Q, Fu J, et al. Stage-specific PET radiomic prediction model for the histological subtype classification of non-small-cell lung cancer. Cancer Manag Res. 2021;13:307-317. doi:10.2147/CMAR.S287128

50. Trebeschi S, Drago SG, Birkbak NJ, et al. Predicting response to cancer immunotherapy using non-invasive radiomic biomarkers. Ann Oncol. 2019;30:998-1004. doi:10.1093/annonc/mdz108

\section{Publish your work in this journal}

Cancer Management and Research is an international, peer-reviewed open access journal focusing on cancer research and the optimal use of preventative and integrated treatment interventions to achieve improved outcomes, enhanced survival and quality of life for the cancer patient.
The manuscript management system is completely online and includes a very quick and fair peer-review system, which is all easy to use. Visit http://www.dovepress.com/testimonials.php to read real quotes from published authors. 